\title{
Pitfalls and limitations in measuring and interpreting the outcomes of mitral valve repair
}

\author{
David H. Adams, MD, and Anelechi C. Anyanwu, MD, MSc, FRCS
}

See related article on page 565 .

From the Department of Cardiothoracic Surgery, Mount Sinai Medical Center, New York, NY.

Received for publication Nov 17, 2005; accepted for publication Nov 28, 2005.

Address for reprints: David H. Adams, MD, Professor and Chairman, Department of Cardiothoracic Surgery, 1190 Fifth Avenue, New York, NY 10029 (E-mail: david. adams@mountsinai.org).

J Thorac Cardiovasc Surg 2006;131:523-9

$0022-5223 / \$ 32.00$

Copyright (C) 2006 by The American Association for Thoracic Surgery

doi:10.1016/j.jtcvs.2005.11.033

I $\mathrm{n}$ this edition of the journal, Serri and colleagues ${ }^{1}$ present their midterm results of valve repair for ischemic mitral regurgitation. They observed significant mitral regurgitation in over half of their patients during the follow-up period, which averaged 28 months, and implied the need for alternative repair techniques or more mitral valve replacement. This is in line with other recent studies that have also shown a high rate of recurrence of mitral regurgitation after annuloplasty for ischemic mitral regurgitation. ${ }^{2-5}$ Can this justification be reliably drawn? Are current approaches to repair ineffective in a significant number of patients with ischemic mitral regurgitation? Can their results be extrapolated to other centers undertaking repair of ischemic mitral regurgitation? We would argue that the study of Serri and colleagues ${ }^{1}$ displays several common clinical and methodologic pitfalls that limit generalization of results from most studies of mitral valve repair. Below, we outline specific challenges in measuring and reporting outcomes of mitral valve repair. Although we illustrate these using the study of Serri and colleagues, ${ }^{1}$ the pitfalls are not unique to their study and apply to varying degrees in all studies of mitral valve repair. Knowledge of these pitfalls is necessary to improve the quality of outcomes-based research on mitral valve repair, to allow accurate interpretation of these studies, and also to permit useful extrapolation of results.

\section{Defining a Patient Cohort}

Serri and colleagues ${ }^{1}$ defined ischemic mitral regurgitation as "mitral regurgitation resulting from prior myocardial infarction associated with normal mitral valve leaflets and chordae." This is an etiologic definition that fails to take into account the valve dysfunction or the lesions that actually result in the mitral regurgitation. What is a "normal mitral leaflet"? Carpentier's pathophysiologic triad describes the interrelationship between leaflet motion (dysfunction), lesions, and etiology. ${ }^{6} \mathrm{Car}-$ pentier's classification of dysfunction is based on the opening and closing motions of the mitral leaflets in relation to the annular plane. Most patients with ischemic mitral regurgitation have type IIIb leaflet dysfunction, with restricted leaflet motion in systole. Wall motion abnormalities, associated with left ventricular dilatation and lateral or apical papillary muscle displacement, result in this type of valve dysfunction. The lesion associated with type IIIb dysfunction is primarily leaflet tethering, especially the posterior-medial scallop of the posterior leaflet (P3). Associated annular dilatation may be present, but leaflet tethering is considered the primary lesion. Type I dysfunction (normal leaflet motion) with isolated annular dilatation is less common but may occur in the setting of basal myocardial infarction. Some patients with ischemic mitral regurgitation have type II (excess leaflet motion) dysfunction. The lesions resulting in type II ischemic mitral regurgitation include an acutely ruptured papillary muscle or a previously infarcted fibrotic and elongated papillary muscle. In rare instances, isolated chordal rupture can also be seen following papillary tip ischemia.

We advocate a more uniform methodology to classify mitral valve cohorts not only on the basis of etiology but inclusive of dysfunctions and lesions. The importance of such an approach is well demonstrated in ischemic mitral regurgitation. The majority of patients have type IIIb dysfunction, and a remodeling annuloplasty must be undersized to overcorrect the septal-lateral dimension and achieve an adequate surface of leaflet coaptation. Patients with ischemic mitral regurgitation 
and leaflet restriction (type IIIb dysfunction) are more likely to have residual leaks compared with patients with type I dysfunction who have normal leaflet motion and are treated in a similar fashion. Furthermore, patients with ischemic mitral regurgitation and type II dysfunction have a better long-term prognosis than those with type IIIb dysfunction. ${ }^{7}$ Thus it is inadequate to describe a population of patients with ischemic mitral regurgitation solely on the basis of etiology. This point is equally valid in patients with a "degenerative" etiology of mitral valve disease. Prolapse (type II dysfunction) of the posterior leaflet due to a single ruptured chord (isolated lesion) has a markedly different repair rate and long-term freedom from recurrent mitral regurgitation compared with bileaflet prolapse with chordal rupture and/or elongation involving multiple leaflet segments. Definition of a population of mitral valve patients on the basis of etiology, dysfunction, and lesions is necessary to allow adequate comparison of outcomes, as all can impact clinical results.

\section{Variations in Surgical Techniques}

To extrapolate the efficacy of a surgical technique in a current article to contemporary practice, it is important that the reader determine whether the technique under evaluation was applied in a standardized and systematic manner that upholds current principles and understanding of pathophysiology. This is particularly relevant in mitral valve repair surgery, as the quality of the result judged by residual or recurrent mitral regurgitation is largely dependent on the manner in which the repair has been performed. For example, it is now well accepted that in the setting of type IIIb dysfunction, patients with ischemic mitral regurgitation should receive an undersized ring to overcorrect the septallateral dimension and ensure an adequate surface of coaptation between tethered leaflets. ${ }^{8,9}$ As Serri and colleagues ${ }^{1}$ do not appear to have undertaken valve sizing in any standardized or logical fashion, their results cannot define the current efficacy of ischemic mitral valve repair. Their stated approach to sizing "that men or large patients tended to receive a $28 \mathrm{~mm}$ ring while women or smaller patients tended to receive a $26 \mathrm{~mm}$ ring" does not have a scientific foundation and indeed is not borne out by their own data, as a third of the patients received neither of these ring sizes. Furthermore, although the authors state rings were undersized, $2 \%$ got a $34-\mathrm{mm}$ ring and $7 \%$ a $32-\mathrm{mm}$ ring - this implies that in $9 \%$ of cases the true size was at least $34 \mathrm{~mm}$. Such a large anterior leaflet surface area would be unusual in patients with IIIb dysfunction and suggests that either the rings may not have been sized appropriately or the etiology was not ischemic. Because it is now widely accepted that use of an appropriately downsized ring is the single most important aspect of mitral repair for ischemic mitral regurgitation, the absence of a precise sizing technique raises doubts regarding surgical approach and could explain their suboptimal long-term results.

The use of incomplete flexible posterior annular bands (in $10 \%$ of patients) further complicates interpretation of their results. Recent articles have linked flexible posterior bands with significant recurrent ischemic mitral regurgitation. ${ }^{2,4}$ It is now well established that patients with ischemic mitral regurgitation can develop dilatation of the anterior annulus between the trigones. ${ }^{10,11}$ Because posterior flexible bands do not correct intertrigonal dilatation, they may not effectively ensure restoration of an adequate surface of leaflet coaptation. In combination with an inconsistent methodology for ensuring proper downsizing of all of the prostheses used in this study (whether partial band or complete ring), these issues raise a significant concern that varied surgical approaches contributed to the suboptimal results.

\section{What Are Acceptable Early Results for Mitral Valve Repair?}

A good long-term outcome follows a low operative mortality (patients have to survive to experience a long-term outcome) and a satisfactory early surgical result (an operation not successful in the short term will remain unsuccessful). Only series with good early results optimally define the long-term efficacy of a surgical procedure. Suboptimal early results in a cohort of mitral valve repairs suggests that adequate operations have not been performed; it is not ideal to define long-term outcomes of repair from such a series. There are presently no guidelines that define standards for an acceptable valve repair. Differing quality and standards for mitral valve repair in different institutions impact longterm outcomes, making the literature even more difficult to interpret. This differs from other forms of valve surgery where the prosthesis, rather than the surgical technique itself, is the primary determinant of long-term outcome.

Residual mitral regurgitation. Significant residual mitral regurgitation following valve repair, documented either on postbypass transesophageal echocardiography (TEE) or predischarge transthoracic echocardiography, implies an immediate failure of the technique employed. In such cases the valve repair was really never successful, and it is not surprising that regurgitation persists into the long term. It would be incorrect to label such cases as "recurrent" mitral regurgitation in studies of long-term outcomes, as the mitral regurgitation was never eliminated at surgery. In the same way that recurrent cancer can only be defined when the primary tumor was successfully and completely resected, recurrent mitral regurgitation should be defined only when surgery initially eliminated the mitral regurgitation. These definitions are often not clarified, and residual mitral regurgitation may account for a substantial proportion of "recurrent" mitral regurgitation reported after ischemic mitral valve repairs. For example, Hung and colleagues ${ }^{5}$ stud- 
ied 30 patients with "recurrent" mitral regurgitation after annuloplasty for ischemic mitral regurgitation in an attempt to understand the mechanisms of recurrence, yet $30 \%$ of these patients had moderate or severe mitral regurgitation on the early postoperative study and should have been categorized with residual mitral regurgitation.

Surgeons generally should not accept any degree of mitral regurgitation other than trivial or mild on the postbypass TEE, as the freedom for need of reoperation, reverse ventricular remodeling, and resulting improvements in quality of life are at least in part dependent on elimination of mitral regurgitation. Significant degrees of residual mitral regurgitation (moderate to severe mitral regurgitation and sometimes even mild mitral regurgitation) should generally trigger a second bypass run and corrective action (for example, further ring downsizing, leaflet patch extension, correction of unrecognized prolapse, or valve replacement). This need for a second bypass run should be uncommon and probably not exceed 5\% as mitral regurgitation should be eliminated in most patients with correct ring choice and sizing. Only in exceptional circumstances should patients leave the operating room with residual moderate mitral regurgitation; such as when a valve is deemed irreparable and nonreplaceable (eg, massive atrial/annular calcification) or when the risks of a second bypass run are thought to outweigh the benefits. In the study by Serri and colleagues, ${ }^{1}$ $10.6 \%$ of patients had moderate mitral regurgitation on the postbypass TEE; all were left uncorrected. The authors did not clarify the reason for the high incidence of residual moderate mitral regurgitation and why it was left uncorrected. A 10\% residual mitral regurgitation rate for ischemic mitral valve repair suggests a problem with the repair technique as moderate mitral regurgitation immediately postbypass should be uncommon. For example, Glower and colleagues had only 1 patient with more than mild mitral regurgitation on the postbypass TEE in a series of 141 repairs for ischemic mitral regurgitation using downsized semirigid complete rings. ${ }^{12}$ If patients leave the operating room with significant mitral regurgitation, then the repair has failed by definition and therefore will also fail in the long term. Indeed, of the 7 patients in the current series who had moderate residual mitral regurgitation, 4 either died or required mitral valve replacement in the early postoperative phase. ${ }^{1}$ Any study with a high degree of residual mitral regurgitation may be relied on to define perioperative but not long-term outcomes of mitral valve repair.

Adverse events (mortality and morbidity). Although combined mitral valve surgery and coronary artery bypass was historically associated with high morbidity and mortality, contemporary series from expert centers report mortality rates in the range of $4 \%$ to $7 \% .{ }^{12-14}$ The operative mortality reported by Serri and associates ${ }^{1}$ of $12.3 \%$ is somewhat higher than other recent series, and although the authors describe their early postoperative results as "very good," it is important to emphasize that in a contemporary practice experienced in mitral valve repair, mortality rates in the region of $5 \%$ should be attainable in patients with ischemic mitral regurgitation.

Indicators of quality. Early results after mitral valve surgery are probably an indirect indicator of the quality of a repair program. Markers of quality include mortality, morbidity, and echocardiographic indicators of successful repair (such as lack of residual mitral regurgitation). The better the early results, the more likely it is that mitral repair has been effectively applied and the greater likelihood that repairs will be durable and effective in the long term. Deficient early results may partly be an indication that repair techniques have not been applied optimally; if that is the case, then long-term results too will be compromised. Indeed, indicators of quality of mitral valve repair appear interrelated, such that performance on one indicator is likely to transform to similar performance on all fronts. For example, the early results by Serri and colleagues ${ }^{1}$ are somewhat compromised by both a high residual mitral regurgitation rate and relatively high mortality, and later follow-up found significant recurrent mitral regurgitation and no reverse remodeling of the left atrium or left ventricle. The interrelation of quality indicators is also well illustrated by the series of Bax and colleagues, ${ }^{15}$ who stringently downsized complete semirigid rings in 51 patients with ischemic mitral regurgitation and reported a $5.6 \%$ operative mortality, $84 \%$ survival at 2 years, significant reduction in left ventricular and left atrial dimensions at 2 years, and no residual or recurrent moderate mitral regurgitation. Thus, consistent practice and good early outcomes are more likely to transform to a sustainable repair. Requisite to defining the benchmark for long-term outcome of mitral repair should therefore be a good performance on early quality indicators and a practice that reflects contemporary concepts in the literature (ring type used, median ring size, residual mitral regurgitation rate, operative mortality).

\section{Incomplete Echocardiographic Data}

Common to almost all studies of mitral valve repair is incomplete availability of echocardiographic data. Complete echocardiographic data at the key time points (preoperative, postbypass, predischarge, early postoperative, and late postoperative) is the exception rather than the rule. In a series of 585 ischemic mitral valve repairs between 1985 and 2002 reported by McGee and colleagues ${ }^{2}$ from Cleveland Clinic Foundation (the specialty leader in outcomesbased research), preoperative echocardiograms were not available for review in $18 \%$ of patients, and postoperative studies were unavailable in $25 \%$ of patients. For most centers an even greater proportion of unavailable echocardiograms is likely. Probably prompted by the paucity of good 
echocardiogram data, several studies on repair for ischemic mitral regurgitation use mortality or reoperation as outcomes, but survival is a weak surrogate for recurrent mitral regurgitation, as is the reoperation rate.

The lack of complete echocardiographic data makes it more difficult to draw conclusions about successfulness of mitral valve repair. In the study by Serri and colleagues, ${ }^{1}$ the paucity of echo data forced the authors to combine postbypass TEE and predischarge echocardiography as a single measurement. It is well established that intraoperative TEE may underestimate mitral regurgitation because of unloading related to anesthesia. ${ }^{16,17}$ Predischarge studies are therefore preferable in establishing a postrepair baseline echocardiogram. Serri and colleagues ${ }^{1}$ also grouped all midterm echocardiograms without incorporating the varying time periods to recurrence of mitral regurgitation. Indeed, only 18 patients were included in the detailed echocardiographic analysis, a subset too small to allow useful extrapolation. Combining the data in this form is often necessary due to high levels of missing or incomplete data but reduces the validity of the results.

Problems with echocardiographic data will likely persist in future reports, as in many institutions, costs, and logistics make it impractical to obtain regular follow-up echocardiography for all patients. Nonetheless, longitudinal studies that include regular echocardiography follow-up will be the gold standard to define the true efficacy of mitral valve repair procedures and should be pursued.

\section{Methodologic Limitations}

\section{Low Case Volume and Long Study Periods}

In most centers, surgery for ischemic mitral regurgitation represents only a small percentage of the overall cardiac surgery. Due to low case volume, sufficient numbers for analysis can only be achieved if studies span long time periods. Even well-established valve centers may have difficulty accruing large numbers of ischemic mitral regurgitation patients, as reflected in the current series reported by Serri and colleagues, ${ }^{1}$ which averaged about 11 ischemic mitral repairs per year. Reports spanning long periods (in the case of Serri and associates, ${ }^{1}$ from 1996 to 2002), which include evolution in surgical understanding, experience, or technique, necessarily produce biased results. Cases done early in the series (when the techniques and protocols have not been mastered and perfected) are more prone to suboptimal results, yet are those more likely to have the longest follow-up. The long-term follow-up therefore often reflects an earlier (learning) era rather than a contemporary (established) one. This is less important for well-established techniques like mitral valve replacement but may significantly bias reports of evolving techniques such as valve repair in ischemic mitral regurgitation.
Bias due to combination of different eras is well illustrated by the study of McGee and colleagues. ${ }^{2}$ Among their findings was an observation that there was no difference in recurrent mitral regurgitation rates between the CarpentierEdwards Classic complete semirigid ring (Edwards Lifesciences, Irvine, Calif), and the Cosgrove-Edwards flexible band (Edwards Lifesciences). Aside from the changes in medical science and perioperative care over the 17 year study period, a notable source of bias is that choice of annuloplasty ring in that series was heavily dependent on era, as was the approach to downsizing. Early in the series, most patients received Carpentier-Edwards Classic rings and from 1997 most patients received Cosgrove-Edwards bands. As the importance of downsizing was not appreciated until the late 1990s, there was no systematic downsizing of the Carpentier-Edwards subgroup, whereas most Cosgrove-Edwards bands were undersized (the median ring size was $30 \mathrm{~mm}$ for Carpentier-Edwards rings compared with $26 \mathrm{~mm}$ for Cosgrove-Edwards bands). Their analysis therefore favors the Cosgrove-Edwards subgroup because they were performed in a more recent era, hence benefiting from modern approaches to management, and also had a specific therapy for ischemic mitral regurgitation (downsizing) that was absent in the Carpentier-Edwards group. Conversely their observation that size of annuloplasty was not predictive of recurrence of mitral regurgitation was confounded because larger rings were generally complete and semirigid and smaller rings were generally flexible band. The inconsistent use of downsized complete semirigid rings probably resulted in underestimation of the long-term efficacy of annuloplasty for ischemic mitral regurgitation in that study.

This problem of a long heterogeneous study period is common to most studies of valve repair and results in underestimation of the efficacy of repair. The problems of low case volume and era-associated knowledge and practice differences can be partly overcome by pooling multicenter data over a short period of time.

Retrospective study design. Most clinical cardiac surgical research is understandably based on retrospective data. ${ }^{18}$ A retrospective study design per se is not a major limitation; indeed, most of the evidence on which we base our practice is derived from such studies. Mitral valve repair, however, presents unique circumstances that limit the usefulness of this study method. Much of the information crucial to understanding mitral valve repair outcomes can only be obtained prospectively. These include the decision to repair or replace, the planned or attempted (as opposed to the completed) repair, the basis for the chosen repair method, specific intraoperative descriptions of valve lesions and dysfunction, the basis for selection and sizing of a ring, echocardiographic measurements specific to the study's focus, and decisions to accept or reoperate for residual mitral 
regurgitation. Such data are important for examining outcomes of mitral valve repair and generally are not obtainable from retrospective chart review. The variations in mitral pathology, valve dysfunction, clinical decision making, and operative approaches are such that retrospective review of operative notes will often be inadequate for studies of valve repair.

Most institutions should now have the database infrastructure to prospectively capture critical perioperative data. Centers with an interest in mitral repair should broaden their databases, or create specific mitral databases, to include relevant data on mitral valve echocardiography, pathology, dysfunction, and surgical repair. Only retrospective analysis of prospectively collected data (that are entered into a database at time of surgery), or planned prospective studies, can provide the most robust data on mitral valve repair.

Evolving technology. Another problem highlighted by Serri and associates' study ${ }^{1}$ is the difficulty in assessing evolving technologies. Although Serri and colleagues do not allude to this, and have presented their data in a manner suggesting that all patients received the same treatment over the study period, in reality this could not have been the case as the understanding and techniques of repair for ischemic mitral regurgitation evolved considerably over the study period. For example, the approach to annuloplasty downsizing is unlikely to have been consistent. Diodato and colleagues ${ }^{14}$ reporting on outcomes of ischemic mitral regurgitation, noted significant changes in practice over a similar period; notably, the practice of downsizing evolved from only $21 \%$ of annuloplasty rings sized $28 \mathrm{~mm}$ and below in 1996 to 1997 to $95 \%$ sized $28 \mathrm{~mm}$ and below by 2000 to 2001. Such evolving patterns complicate analysis and interpretation of data as patients have not received the same treatment. Several new annuloplasty devices and modifications of surgical technique have emerged in the last 5 years and continue to be developed ${ }^{9,19}$; depending on the usage of such new technologies (some are beneficial and some detrimental), impact on outcome could be substantial. Investigators should accommodate for these changes in practice such as by analyzing changes in outcome over time (as most practice changes are temporally linked) or by performing subgroup analysis to specifically examine the impact of new devices and techniques. Changes are, however, often multiple and are not applied in a systematic fashion, making analysis and logical deduction difficult. Multicenter studies with standardized protocols conducted over a relatively short time period may minimize the effect of evolving technology; however, as midterm results of such studies are presented, the techniques themselves may have become obsolete, due to subsequent arrival of new technologies and improved understanding.

Statistical limitations. Analysis of valve-related outcomes presents specific statistical quandaries because of difficulty in depicting and analyzing time-related change where there are several competing outcomes (such as death, valve reoperation, and transplantation). This is further complicated where the outcome is graded (mild, moderate, or severe regurgitation) rather than binary and may change over time (to lesser or greater degrees of mitral regurgitation). These issues are well discussed elsewhere, notably by Grunkemeier and associates ${ }^{20,21}$ and Blackstone. ${ }^{22}$ Obstacles to statistical analysis also arise because postoperative data (echocardiography in this case) on mitral valve repair are often derived from clinical assessments made at irregular intervals for reasons that differ from patient to patient. In some patients, death, disability, relocation, reoperation, transplantation, or a number of nonrandom events (possibly related to the valve disease or valve repair) may interrupt obtaining further measurements. A patient who does not have any echocardiogram after the first year may well be bedridden in a nursing home with severe heart failure but will be assumed to have a (censored) good outcome. Standard techniques based on censoring may therefore be flawed because censoring is assumed to be noninformative (ie, the loss to follow-up bears no relationship to the intervention or outcome). Authors of valve outcome studies have used various ways to handle this and generally attempt to oversimplify what is actually a complicated stream of data. For example, Serri and colleagues ${ }^{1}$ analyzed postoperative echocardiographic data as though they were obtained at the same time, grouping together follow-up echocardiograms obtained at any interval from several months to several years after surgery. They consolidated these into a single figure for the incidence of "midterm" mitral regurgitation. How does one interpret a figure of $57 \%$ moderate to severe recurrent mitral regurgitation in the midterm-is that at 1 year, 3 years, 5 years? This figure also formed the basis of multivariate analysis for predictors of postoperative mitral regurgitation. Such analysis is heavily flawed as occurrence of mitral regurgitation is a time-related event; mitral regurgitation at 1 year is substantially different from that occurring at 7 years and thus cannot be combined as though they are one. How does one deal with long gaps in assessment, such as a patient who had no mitral regurgitation on a 1-year study and presents with symptoms 4 years later with echocardiogram showing severe mitral regurgitation? Although easiest to assume that mitral regurgitation recurred at the point of echocardiogram, in reality it may have occurred several years earlier.

Even where the outcome measure does not suffer from irregular and inconsistent measurement intervals (such as freedom from valve reoperation), conventional actuarial methods overestimate the true frequency of valve-related events as they assume patients remain at risk of valve failure after death (as observations are censored at death). ${ }^{21}$ Some of these problems can be overcome with advanced statistical 
methods that are generally beyond the scope of the clinical surgeon. These require specialized statistical expertise. ${ }^{22}$ The problem, however, remains that with most valve outcome studies authors prefer to oversimplify the outcomes, in a manner that they can easily analyze and interpret, and in doing so invariably report inaccurate or inadequate results. As Blackstone ${ }^{22}$ puts it "Unfortunately, we insist on doing our own statistics, content to live with the simple answers rather than those that are most revealing or most accurate."

Serri and colleagues ${ }^{1}$ also made a significant statistical error in their presentation and analysis, which highlights the difficulty in using mitral regurgitation grade as an outcome. In their data summary and analysis, they have treated the mitral regurgitation grade as a continuous variable, deriving means, standard deviations, and also using linear regression to predict recurrent mitral regurgitation. Mitral regurgitation grade is not, however, a continuous variable but rather a categorical variable (or more specifically an ordinal or rankordered variable) and therefore cannot be subjected to mathematical manipulation. The 4 grades could have just as easily been labeled I, II, III, and IV or trivial, mild, moderate, and severe. The numerical assignments of 1 to 4 are arbitrary and made just to illustrate the rank order, rather than as a quantification or direct measurement of the mitral regurgitation (grade 4 mitral regurgitation is not twice grade 2 mitral regurgitation). They have also incorrectly presented the New York Heart Association (NYHA) class as a continuous variable by summarizing it as a mean and standard deviation. Data such as NYHA class and mitral regurgitation grade should be summarized using medians or frequency and analyzed as categorical or ordinal variables. The lack of a quantitative description of mitral regurgitation that can be uniformly reported on a continuous scale poses difficulty in analysis. Authors often resort to categorizing all mitral regurgitation into 2 groups with no discrimination based on severity of mitral regurgitation (freedom from recurrent mitral regurgitation being freedom from moderate or severe mitral regurgitation). Echocardiographic quantification of mitral regurgitation using a numerical rather than qualitative description of mitral regurgitation would greatly simplify data analysis.

\section{Conclusions}

The clinical practice of valve repair presents unique problems that complicate measurement and analysis of outcomes. Although we have illustrated this using repair for ischemic mitral regurgitation as presented by Serri and colleagues, ${ }^{1}$ the same applies to other valve repair surgery. Although it is true that all valve repairs have a long-term failure rate, most published series have pitfalls that prevent accurate quantification of this rate. To determine the efficacy of any mitral repair operation, surgical teams undertaking studies should have a thorough understanding of pathophysiology and apply techniques in a standardized fashion. Centers undertaking outcome efficacy studies should have low postoperative mortality and a documented low residual mitral regurgitation rate and should be well versed and specialized in mitral repair. To allow later meaningful analysis, data on all valve procedures should be collected prospectively in clinical databases and should include relevant perioperative and scheduled postoperative echocardiographic findings and measurements. Period of recruitment for these studies should be relatively short. Collaborative efforts between different centers are one way to ensure meaningful recruitment over a period of months rather than years. At least some valve centers should strive to obtain scheduled periodic follow-up quantitative echocardiography on all patients, as this would markedly simplify analysis and interpretation of results. Where the follow-up data are complex with several repeated measures, high loss to follow-up, or competing outcomes, authors should apply specialized statistical techniques where indicated rather than attempt simplified analysis of data, which will invariably be erroneous. Only the results of such studies can define, with relative certainty, the long-term outcomes of mitral valve repair for ischemic mitral regurgitation or any other mitral disease.

\section{References}

1. Serri K, Bouchard D, Derners P, Coutu M, Pellerin M, Carrier M, et al. Is good perioperative echocardiographic result predictive of durability in ischemic mitral valve repair. J Thorac Cardiovasc Surg. 2006;131:565-73.

2. McGee EC, Gillinov AM, Blackstone EH, Rajeswaran J, Cohen G, Najam F, et al. Recurrent mitral regurgitation after annuloplasty for functional ischemic mitral regurgitation. J Thorac Cardiovasc Surg. 2004;128:916-24.

3. Tahta SA, Oury JH, Maxwell JM, Hiro SP, Duran CM. Outcome after mitral valve repair for functional ischemic mitral regurgitation. J Heart Valve Dis. 2002;11:11-8.

4. Bhudia SK, McCarthy PM, Smedira NG, Lam BK, Rajeswaran J, Blackstone EH. Edge-to-edge (Alfieri) mitral repair: result in diverse clinical settings. Ann Thorac Surg. 2004;77:1598-606.

5. Hung J, Papakostas L, Tahta SA, Hardy BG, Bollen BA, Duran CM, et al. Mechanism of recurrent ischemic mitral regurgitation after annuloplasty: continued LV remodeling as a moving target. Circulation. 2004;110(Suppl 1):II85-90.

6. Carpentier A. Cardiac valve surgery-the "French correction." J Thorac Cardiovasc Surg. 1983;86:323-37.

7. Gillinov AM, Wierup PN, Blackstone EH, Bishay ES, Cosgrove DM, White $\mathrm{J}$, et al. Is repair preferable to replacement for ischemic mitral regurgitation? J Thorac Cardiovasc Surg. 2001;122:1125-41.

8. Badhwar V, Bolling SF. Mitral valve surgery in the patient with left ventricular dysfunction. Semin Thorac Cardiovasc Surg. 2002;14:133-6.

9. Filsoufi F, Salzberg SP, Adams DH. Current management of ischemic mitral regurgitation. Mt Sinai J Med. 2005;72:105-15.

10. Heub AC, Jatene FB, Moreira LF, Pomerantzeff PM, Kallas E, de Oliveira SA. Ventricular remodeling and mitral valve modifications in dilated cardiomyopathy: new insights from anatomic study. $J$ Thorac Cardiovasc Surg. 2002;124:1216-24.

11. Ahmad RM, Gillinov AM, McCarthy PM, Blackstone EH, AppersonHansen C, Qin JX, et al. Annular geometry and motion in human ischemic mitral regurgitation: novel assessment with three-dimensional echocardiography and computer reconstruction. Ann Thorac Surg. 2004; 78:2063-8. 
12. Glower DD, Tuttle RH, Shaw LK, Orozco RE, Rankin JS. Patient survival characteristics after routine mitral valve repair for ischemic mitral regurgitation. J Thorac Cardiovasc Surg. 2005;129:860-8.

13. Gillinov AM, Blackstone EH, Rajeswaran J, Mawad M, McCarthy PM, Sabik JF, III, et al. Ischemic versus degenerative mitral regurgitation: does etiology affect survival? Ann Thorac Surg. 2005;80:811-9.

14. Diodato MD, Moon MR, Pasque MK, Barner HB, Moazami N, Lawton JS, et al. Repair of ischemic mitral regurgitation does not increase mortality or improve long-term survival in patients undergoing coronary artery revascularization: a propensity analysis. Ann Thorac Surg. 2004;78:794-9.

15. Bax JJ, Braun J, Somer ST, Klautz R, Holman ER, Versteegh MI, et al. Restrictive annuloplasty and coronary revascularization in ischemic mitral regurgitation results in reverse left ventricular remodeling. Circulation. 2004;110:II103-8.

16. Konstadt SN, Louie EK, Shore-Lesserson L, Black S, Scanlon P. The effects of loading changes on intraoperative Doppler assessment of mitral regurgitation. J Cardiothorac Vasc Anesth. 1994;8: $19-23$.
17. Aklog L, Filsoufi F, Flores KQ, Chen RH, Cohn LH, Nathan NS, et al. Does coronary artery bypass grafting alone correct moderate ischemic mitral regurgitation? Circulation. 2001;104(Suppl 1):I68-75.

18. Anyanwu AC, Treasure T. Surgical research revisited: clinical trials in the cardiothoracic surgical literature. Eur j Cardiothorac Surg. 2004; 25:299-303.

19. Levine RA, Schwammenthal E. Ischemic mitral regurgitation on the threshold of a solution: from paradoxes to unifying concepts. Circulation. 2005;112:745-58.

20. Grunkemeier GL, Jamieson WR, Miller DC, Starr A. Actuarial versus actual risk of porcine structural valve deterioration. $J$ Thorac Cardiovasc Surg. 1994;108:709-18.

21. Grunkemeier GL, Wu Y. Interpretation of nonfatal events after cardiac surgery: actual versus actuarial reporting. J Thorac Cardiovasc Surg. 2001;122:216-9.

22. Blackstone EH. Breaking down barriers: helpful breakthrough statistical methods you need to understand better. J Thorac Cardiovasc Surg. 2001;122:430-9. 